\title{
Time Varying Filters for Estimation of Evoked Potentials
}

\author{
G. Sita, R. V. S. Sastry and A. G. Ramakrishnan
}

Biomedical Engineering Laboratoly, Department of Electrical Engineering, Indian Institute of Science, Bangaloire -- 560012

\section{ABSTRACT}

This paper presents a method for the estimation of Evoked Potentials (EP) using an a posteriori time varying filter based on Discrete Wavelet Transform (DWT). The proposed filter uses Alternate Subensemble Averaging (ASA) to arrive at the signal and noise scalogram estimates. This filter, when applied on the Ensemble Average (EA), leads to an improvement in the SNR. When applied on the individual trials, the ongoing EEG is significantly eliminated, revealing the underlying EP. The proposed filter has been tested on simulated data.

Keywords : Evoked Potential, Estimation, Discrete Wavelet Transform, Wiener filtering, time varying filtering

\section{INTRODUCTION}

In order to observe transient events in the neural pathways, it is essential that an EP estimate is obtained in as few stimulus repetitions as possible. However, ensemble averaging requires a large number of stimuli for good noise suppression. So, techniques for filtering the EA or individual trials are being developed [1]. Earlier attempts [2] used the Wiener filter, estimated a posteriori from an ensemble of $\mathbf{P}$ single trials based on the expression given below:

$\hat{H}(w)=\frac{\hat{\phi}_{s s}(w)}{\hat{\phi}_{s s}(w)+\frac{1}{P} \hat{\phi}_{n s}(w)}=\frac{P}{P-1}\left(1-\frac{1}{P} \frac{\overline{\phi_{y y_{p}}}(w)}{\phi_{\overline{y_{p}}}(w)}\right)$

where $\phi_{\overline{y_{P}}}(\mathrm{~W})$ and $\overline{\phi_{y y_{P}}}(\boldsymbol{w})$ are the power spectrum of the EA and the average of all the elementary power spectra. de Weerd et al [3] suggested estimating the signal and noise spectra based on ASA and time varying filtering based on an octave filter bank, which was nonorthogonal and hence, not energy preserving. The current work uses the multirate formalism for designing an orthogonal Perfect Reconstruction Quadrature Mirror Filter (PR-QMF) bank [4].

\section{METHOD}

The model for EP used in this study is given by,

$$
y_{i}(t)=s(t)+n_{i}(t), i=1, \ldots, P ; \quad 0 \leq t \leq T
$$

where $y_{i}(t)$ is the i-th recorded trial, $s(t)$ is the EP and $n_{i}(t)$ is the zero mean, stationaly random noise, uncorrelated with $s(t)$, which is assumed to be deterministic, transient and independent of the trial. EEG is simulated as the output of a fourth order autoregressive process driven by Gaussian white

Proceedings RC IEEE-EMBS \& 14th BMESI - 1995

3.33 noise. The variance of the noise was scaled to give-an SNR of $-10 \mathrm{~dB}$. An ensemble of fifty trials was used for testing the proposed filter. This was split into $\mathrm{N}$ subensembles, each of size $M=P / N,(M$ even) and an alternate average is computed for each. Considering the transient nature of the $\mathrm{EP}$, it is appropriate to use a time-varying-spectral formulation in the Wiener filter. An efficient method for this is using a linear Time-Frequency distribution, namely, DWT. The orthogonal FR-QMFbank of constant relative bandwidth inherent in the DWT is well matched to the typical time frequency structure of evoked potentials, consisting of early components of relatively short duration, high frequency and large BW and later components of longer duration, low frequency and small BW. So, the DWT is evaluated using Daubechies' 8-tap filter [5] and then, the square of the DWT, known as scalogram, $E_{\widetilde{y}_{M, N}^{(n)}}^{(n, j)}(i)$, is computed for each subensemble average, $\tilde{y}_{M, N}^{(n)},(n=1, \ldots, N)$. All such scalograms are then averaged thus:

$$
\overline{E_{\tilde{y}_{M, N}}}(i, j)=\frac{1}{N} \sum_{n=1}^{N} E_{\tilde{y}_{M, N}}^{(n)}(i, j)
$$

The scalogram of the EA is also computed and the Time VaryingA posteriori Wiener Filter (TVAWF) is given by

$$
H(i, j)=1-\frac{1}{N} \frac{\overline{E_{\tilde{y}_{M, N}}}(i, j)}{E_{\bar{y}}(i, j)}
$$

This expression differs from that of Bertrand [6] in using the ASA for estimation of signal and noise scalograms. The TVAWF, $H(i, j)$, is applied by weighing the DWT of EA to get the final EP estimate.

\section{RESULTS}

A simulaied EP and a typical ensemble element (with added noise) is shown in Fig. (la). Fig. (lb) shows the components of the EP corresponding to the four octave subbands of the DWT, while Fig (lc) depicts their corresponding scalogram. Fig. (2a) shows the EA and the filtered EA for one simulation.. Fig. (2c) shows the result of application of the proposed filter on the individual trials illustrated in Fig.(2b). As can be seen, the filtered responses have a much better consistency in the peak components than the originals. This implies that the ongoing EEG has been significantly eliminated in the filtered responses by TVAWF. 

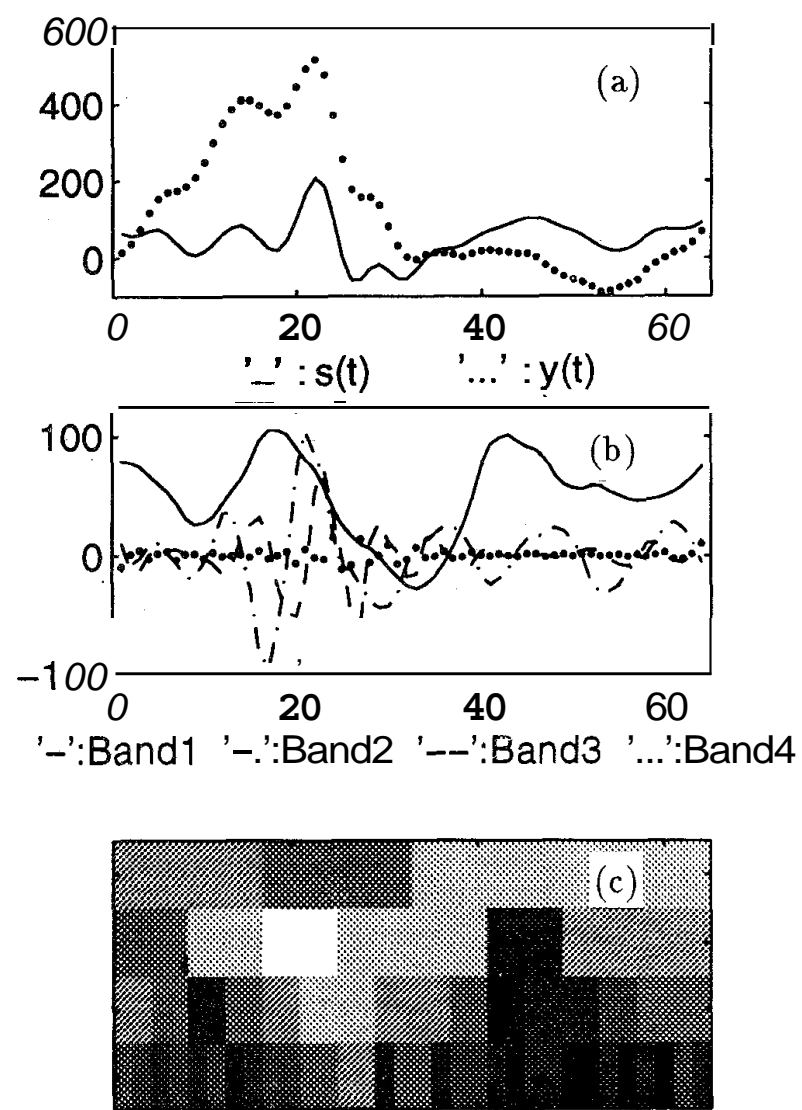

Fig. 1 [a] The simulated SSEP, $s(t)$ and a typical trial, $y(t)$. [b] Components of $s(t)$ corresponding to four octave subbands. [c] Time frequency energy distributionof the sub-band signals in $[\mathrm{b}]$.

\section{CONCLUSION}

Extensive simulation studies have clearly demonstrated the general applicability of the time varying filtering method based on DWT and ASA and its superiority to the conventional a posteriori Wiener filtering when dealing with transient signals. The proposed technique is elegant and computationally simple, since the filter estimation using ASA requires only $N+1$ DWTs to be computed in contrast to $\mathrm{P}+1$ DWTs in Bertrand's scheme [6]. Also, alternate addition and subtraction of subensemble elements is less subject to inhomogenities due to slow variations within the ensemble. That the TVAWF is able to improve the SNR of individual sweeps is significant.

\section{REFERENCES}

1. Yu X H, He Z Y and Zhang Y S (1994) “ Time-Varying Adaptive Filters for Evoked Potential Estimation," $\boldsymbol{Z E F E}$ Trans.Biomed.Eng., 41, 1062-1071.

Proceedings RC IEEE-EMBS \& 14th BMESI - 1995
2. Walter D O(1969) "A posteriori Wiener Filtering of Averaged Evoked Responses," Electroenceph. Clin. Neurophysiol. Suppl. 27, 61-70.

3. de Weerd, J P C (1981) “Aposteriori time-varying filtering of averaged evoked potentials - II. Mathematical and Computational Aspects," Biol. Cybem., 41, 223-234.

4. Vaidyanathan P P (1993) "Multirate Systems and Filter Banks,” Prentice Hall Inc., New Jersey.

5. Daubechies I (1988) "Orthonormal bases of compactly supported wavelets," Comm. Pure Appl. Math., 41, 909-996.

6. Bertrand O, Bohorquez J and Pernier J (1994) "Timefrequency digital filtering based on an invertible wavelet transform: An application to evoked potentials," IEEE Trans. Biomed. Eng., 41, 77-88.
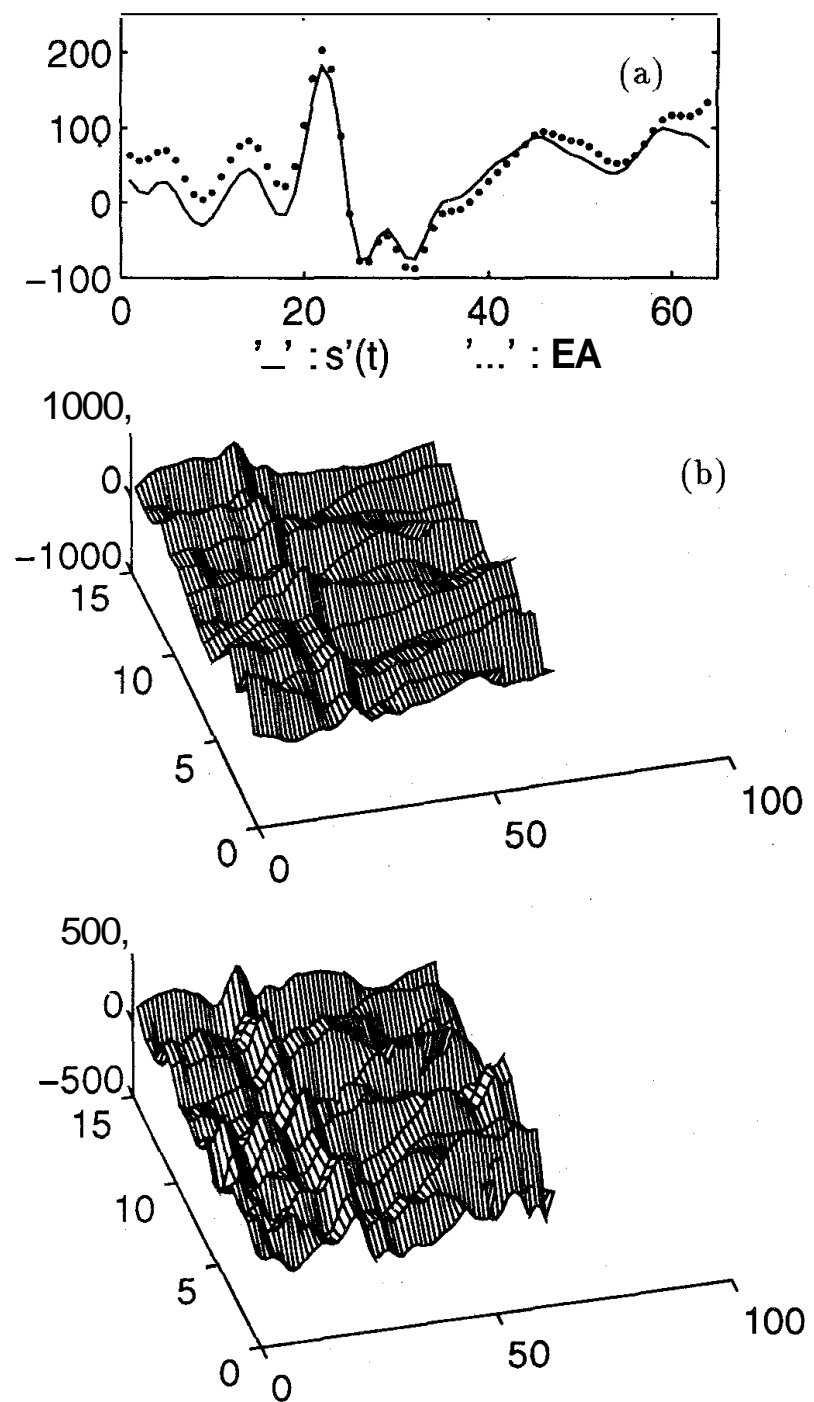

Fig. 2 [a] The EA before and after the application of the TVAWF. [b] Ensemble of EP trials contaminatedby noise. [c] Ensemble after application of TVAWF. 